\title{
Synthesis, Characterization and Optical Constants of Silicon Oxycarbide
}

\author{
Faisal Ahmed Memon ${ }^{1,2, *}$, Francesco Morichetti ${ }^{1}$, Muhammad Ishaque Abro $^{3}$, Giosue Iseni ${ }^{4}$, Claudio Somaschini ${ }^{4}$, Umair \\ Aftab $^{3}$, Andrea Melloni ${ }^{1}$ \\ ${ }^{1}$ Dipartimento di Elettronica, Informazione e Bioingegneria (DEIB), Politecnico di Milano, via Ponzio 34/5, 20133 Milano Italy \\ ${ }^{2}$ Department of Telecommunications Engineering, Mehran University of Engineering \& Technology, Jamshoro 76062, Pakistan \\ ${ }^{3}$ Department of Metallurgy and Materials Engineering, Mehran University of Engineering \& Technology, Jamshoro 76062, Pakistan \\ ${ }^{4}$ Polifab, Politecnico di Milano, via Giuseppe Colombo 81, 20133 Milano Italy
}

\begin{abstract}
High refractive index glasses are preferred in integrated photonics applications to realize higher integration scale of passive devices. With a refractive index that can be tuned between $\mathrm{SiO}_{2}(1.45)$ and a$\mathrm{SiC}$ (3.2), silicon oxycarbide SiOC offers this flexibility. In the present work, silicon oxycarbide thin films from $0.1-2.0 \mu \mathrm{m}$ thickness are synthesized by reactive radio frequency magnetron sputtering a silicon carbide $\mathrm{SiC}$ target in a controlled argon and oxygen environment. The refractive index $n$ and material extinction coefficient $k$ of the silicon oxycarbide films are acquired with variable angle spectroscopic ellipsometry over the UV-Vis-NIR wavelength range. Keeping argon and oxygen gases in the constant ratio, the refractive index $n$ is found in the range from 1.41 to 1.93 at $600 \mathrm{~nm}$ which is almost linearly dependent on RF power of sputtering. The material extinction coefficient $k$ has been estimated to be less than $10^{-4}$ for the deposited silicon oxycarbide films in the visible and near-infrared wavelength regions. Morphological and structural characterizations with SEM and XRD confirms the amorphous phase of the SiOC films.
\end{abstract}

\section{Introduction}

High performance optical waveguide materials must combine low loss with the flexibility to fabricate a variety of passive devices on a single material platform. With a refractive index that can be tuned between silica glass $\mathrm{SiO}_{2}$ (1.45) and amorphous silicon carbide a-SiC (3.2), silicon oxycarbide SiOC offers this flexibility. However, the methods which were used to produce silicon oxycarbide such as chemical vapor deposition (CVD) $[1,2,3,4]$ and sol-gel pyrolysis [5, 6, 7, 8] introduce hydrogen and high material absorption. In the recent years, the authors in $[9,10]$ showed the deposition of SiOC films with RF sputtering technique and discussed physical and structural characteristics of SiOC thin films. In another study by T. Nakai et. al. [11], low refractive-index SiOC films were developed with $\mathrm{rf}$ sputtering and exploited for HD DVD rewritable media. However optical properties ( $n$ and $k$ ) of the SiOC films over a wide wavelength spectrum ranging from UV to near-infrared are not reported. In this work, we demonstrate fabrication of silicon oxycarbide thin films on $\mathrm{Si}$ (100) substrate by reactive $\mathrm{RF}$ magnetron sputtering of a $\mathrm{SiC}$ target in a controlled argon and oxygen gases environment. The SiOC thin films are characterized by variable angle spectroscopic ellipsometry over the broad UV-VIS-NIR wavelength range to obtain optical constants (refractive index and absorption spectra). The motivation to use sputter deposition comes from the fact that it has potential to prepare high quality material with essentially no hydrogen content at room temperature compared to typical deposition techniques such as CVD [3] which introduce hydrogen and high material absorption coefficient $\alpha$ on the order of $10^{5} \mathrm{~cm}^{-1}$. The refractive index $n$ of the deposited SiOC films ranges from 1.41 to 1.93 at $600 \mathrm{~nm}$ as a function of RF power level. The extinction coefficient $k$ of all the deposited SiOC films is estimated to be less than $10^{-4}$ above $\lambda=1000 \mathrm{~nm}$. To the best of our knowledge, it is the first time to report optical properties mainly the refractive index $n$ and extinction coefficient $k$ spectra in a broad wavelength range extending from UV $(300 \mathrm{~nm})$ to Near IR (1200 nm). Profilometry and scanning electron microscopy (SEM) were used to determine thickness and analyse morphology of the deposited SiOC films, respectively. The phase of the films was examined with X-ray diffraction (XRD).

\section{Deposition of SiOC Films}

Depositions of silicon oxycarbide thin films were carried out by reactive RF magnetron sputtering of a $99.9 \%$ pure 3 inches diameter silicon carbide $\mathrm{SiC}$ target which was manufactured by Ajaint International. The sputtering chamber was pumped down to better than $10^{-4} \mathrm{~Pa}$ before the argon and oxygen gas flux. High purity argon $\mathrm{Ar}$ and oxygen $\mathrm{O}_{2}$ were used as the plasma and reactive gases in

\footnotetext{
* Corresponding author: faisalahmed.memon@polimi.it
} 
the sputtering chamber during preparation of amorphous silicon oxycarbide films. The depositions were performed on boron doped p-type Si (100) substrate at room temperature. Prior to deposition, substrates were first cleaned in acetone and then isopropanol in ultrasonic cleaner to remove the contaminants and organic matter on $\mathrm{Si}$ substrate surface. In order to clean the $\mathrm{SiC}$ target surface, fifteen minutes pre-sputtering without reactive gas was done each time before the actual film deposition. To check repeatability and achieve precise control of the sputter process a large number of SiOC samples were produced by setting the recipe: RF power level from 50 to 450 watts, argon to oxygen gas ratio of $47 \mathrm{sccm}$ and deposition time of 15 minutes. Here we report the results of $6 \mathrm{SiOC}$ films which were deposited as function of increasing RF power level.

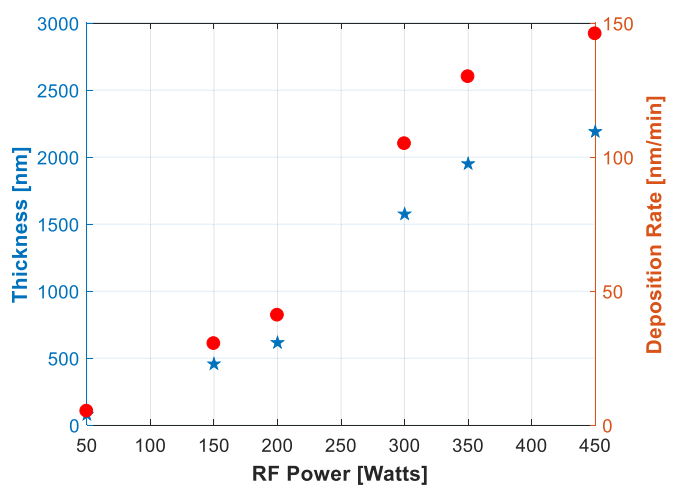

Fig. 1. Blue stars show measured thickness and red circles show deposition rate of the deposited SiOC films as a function of RF power.

The thickness of the SiOC films was measured with KLA Tencor P-15 Surface Profilometer. To determine the thickness with profilometer, kapton tape was used on cleaned substrates as a mask before deposition. The deposited samples were cleaned in acetone by means of ultrasonic device to eliminate the mask. Due to the good quality adhesion of sputtered SiOC films on Si (100) substrates, no wear off or pin holes in SiOC films were observed after ultrasonication. Figure 1 shows the thickness and deposition rate of silicon oxycarbide thin films deposited as a function of RF power from 50 to $450 \mathrm{~W}$. The thickness of the SiOC films was determined to be between $100 \mathrm{~nm}$ and $2000 \mathrm{~nm}$. The deposition rate increases with RF power that is varied from 50 to $450 \mathrm{~W}$ and a large rate of $150 \mathrm{~nm} / \mathrm{min}$ has been achieved, which suggests that micron size films can be prepared in a fraction of an hour.

\section{Characterization of SiOC Films}

The refractive index $n$ and extinction coefficient $k$ dispersion spectra of the SiOC films were measured with variable angle spectroscopic ellipsometer (VASE J.A. Woollam Inc. USA). The measurement angles used were $72^{\circ}$ and $74^{\circ}$ which are commonly adopted in [12]. All the
SiOC samples were characterized over the wide wavelength range from $300-1200 \mathrm{~nm}$ which covered UV - Visible - Near IR regions with spectral sampling rate of $10 \mathrm{~nm}$ and ellipsometric $\psi$ and $\Delta$ data were acquired. A four-layer film/substrate stack comprising surface-roughness, $\mathrm{SiOC}$ thin film, native $\mathrm{SiO}_{2}$ layer, $\mathrm{Si}$ substrate as shown in figure (2) was used as a model for our samples and to extract the optical parameters $n$ and $k$ of the SiOC films. To best fit the data and extract the optical parameters over the entire wavelength range, Tauc-Lorentz (TL) model [13] that is intrinsically Kramers-Kronig [13,14] consistent was used to represent the SiOC film and the obtained extinction coefficient and refractive index values did not require further processing. Jellison and Modine [13] developed the Tauc-Lorentz model which is formulated as,

$$
\begin{aligned}
& \varepsilon_{n_{-} T-L}=\varepsilon_{n 1}+i \varepsilon_{n 2} \quad \text { where } \\
& \varepsilon_{n 2}= \begin{cases}\frac{1}{E} \cdot \frac{A_{n} E o_{n} C_{n}\left(E-E g_{n}\right)^{2}}{\left(E^{2}-E o_{n}^{2}\right)^{2}+C^{2} E^{2}} & \text { for } E>E g_{n} \\
0 & \text { for } E \leq E g_{n}\end{cases} \\
& \varepsilon_{n 1}=\frac{2}{\pi} P \int_{E g_{n}}^{\infty} \frac{\xi \cdot \varepsilon_{n 2}(\xi)}{\xi^{2}-E^{2}} d \xi
\end{aligned}
$$

Equation (1) is the combination of real (2) and imaginary parts (3) of the dielectric function of Tauc-Lorentz oscillator. In equation (2), $A_{n}$ is the strength of the $\varepsilon_{n 2}(E)$ peak in $\mathrm{eV}, \mathrm{C}_{\mathrm{n}}$ is the broadening of the peak in $\mathrm{eV}, \mathrm{Eo}_{\mathrm{n}}$ is the central energy of the peak in $\mathrm{eV}$ and $\mathrm{Eg}_{\mathrm{n}}$ is the bandgap energy in $\mathrm{eV}$. Since $\mathrm{Eg}_{\mathrm{n}}$ is declared as a fit parameter in the TL oscillator to model the ellipsometric $\psi$ and $\Delta$ of the SiOC films, the value does not represent the actual bandgap of the material. The real part $\varepsilon_{n 1}$ (3) of the dielectric function (1) is derived from the expression of $\varepsilon_{\mathrm{n} 2}$ (2) using the Krameres-Kronig integration $[13,14,15]$. In equation (3), $\mathrm{P}$ is the Cauchy principal value containing the residues of the integral at poles located on lower half of the complex plane and along the real axis. $\mathrm{WVASE}^{\circledR}$ [15] uses complete analytical solution to the Kramers-Kronig integral as given in [15].

High resolution images of silicon oxycarbide films cross section were obtained with a LEO 1525 scanning electron microscope (SEM). To avoid electrons charging effect, a thin layer of platinum $(\approx 2 \mathrm{~nm})$ was deposited on SiOC films. The electrons were accelerated at a voltage of $10 \mathrm{kV}$ and the signal was collected from inlens detector keeping the distance around $6 \mathrm{~mm}$ between the SiOC sample and the electron gun. The microstructure of the SiOC films was examined from $\mathrm{x}$ ray diffraction patterns measured over Range $2 \theta 20-50$ degrees with XRD D8 Advance (Bruker Corp).

\section{Results and Discussion}

The optical properties of the silicon oxycarbide films grown on $\mathrm{Si}$ subtstrate as a function of RF power have 
been characterized by spectroscopic ellipsometry. The ellipsometric $\psi$ and $\Delta$ data acquired over the wide wavelength range from 300 to $1200 \mathrm{~nm}$ for each SiOC film have been modelled with a four-layer model comprising surface roughness/Tauc-Lorentz/native$\mathrm{SiO}_{2} / \mathrm{Si}$ layers as illustrated in figure 2 . Since ellipsometry is very sensitive to native silica and surface roughness conditions, the $2 \mathrm{~nm} \mathrm{SiO}_{2}$ and surface roughness layers were added to improve fit and MSE. The optical constants of $\mathrm{Si}$ substrate and $\mathrm{SiO}_{2}$ were used from the $\mathrm{WVASE}^{\circledR}$ database [15]. The surface roughness SR layer based on Bruggemann and Mawxell-Garnett effective medium approximation (EMA) [16] composed of $50 \%$ material and $50 \%$ voids is used to simulate the surface roughness of SiOC films. The SR layer is ellipsometry equivalent of AFM and gives peak-tovalley roughness, not the rms roughness. The parameters of the Tauc-Lorentz oscillator described earlier were declared fit in the model shown in figure 2(a) and regression analysis algorithm was run to minimize the global mean squared error (MSE). Figure 2(b) shows an example of fitting of ellipsometric $\psi$ and $\Delta$ spectra of the SiOC film deposited at $300 \mathrm{~W}$ over the broad wavelength range $300-1200 \mathrm{~nm}$ at one measurement angle $74^{\circ}$ for the sake of brevity.

(a)

\begin{tabular}{|c|}
\hline Surface roughness layer $0-6 \mathrm{~nm}$ \\
\hline SiOC film $100-2000 \mathrm{~nm}$ \\
\hline native $\mathrm{SiO}_{2}$ layer $2 \mathrm{~nm}$ \\
\hline $\mathrm{Si}$ substrate \\
\hline
\end{tabular}

(b)

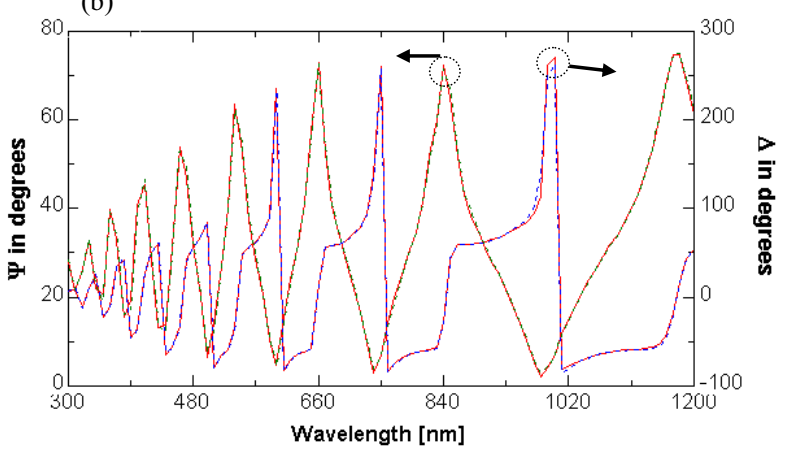

Fig. 2. (a) four-layer stack to model the ellipsometric $\psi$ and $\Delta$ data (b) ellipsometric $\psi$ and $\Delta$ data (green and blue dotted lines) of SiOC film deposited at RF power $300 \mathrm{~W}$ acquired at measurement angle of $72^{\circ}$ and model fit (red solid lines)

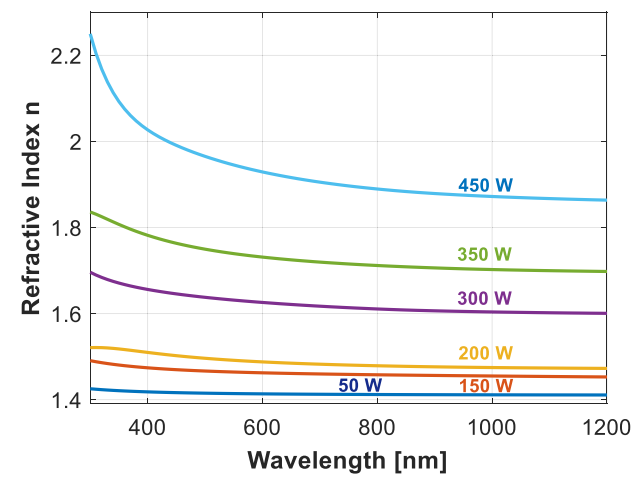

Fig. 3. Refractive index $n$ spectra of SiOC films deposited as a function of RF power $50-450 \mathrm{~W}$

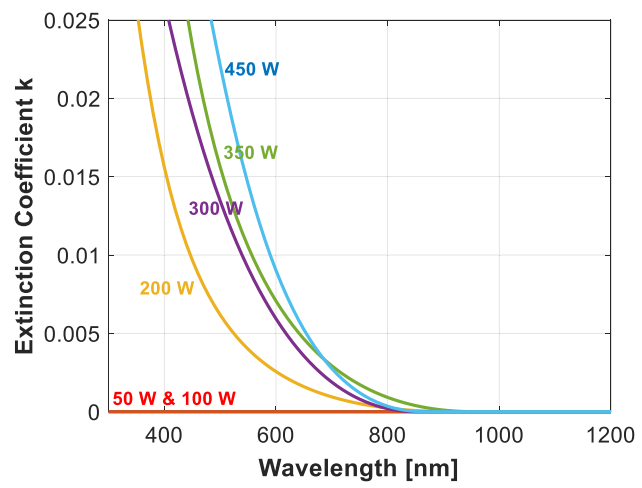

Fig. 4. Extinction coefficient $k$ spectra of $\mathrm{SiOC}$ films deposited as a function of RF power $50-450 \mathrm{~W}$

Figures 3 and 4 show the refractive index $n$ and material absorption (extinction coefficient) $k$ spectra of the SiOC films deposited by increasing RF power level from 50 to $450 \mathrm{~W}$. As shown in figure 3 , the refractive index spectral curves are decreasing as a function of wavelength from UV to Near-IR region which demonstrate a typical dielectric medium behaviour. The refractive index $n$ of the deposited $\mathrm{SiOC}$ films ranges from 1.41 to 1.93 at visible light wavelength $\lambda=600 \mathrm{~nm}$. The extinction coefficient $k$ in all the SiOC films is less than $10^{-4}$ above $\lambda=1000 \mathrm{~nm}$. In the visible region, the SiOC films show better transparency as compared to SiOC films deposited with CVD [3]. The roughness of the SiOC films deposited at RF power level of $450 \mathrm{~W}$ is found to be around $6 \mathrm{~nm}$ by fitting SR layer and it reduces to almost nothing with decreasing RF power level to $50 \mathrm{~W}$. 


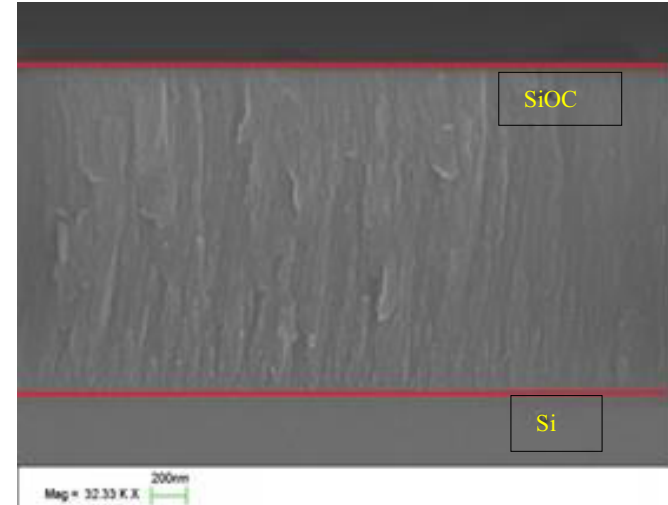

Fig. 5. Cross section image of SiOC film deposited at $350 \mathrm{~W}$ obtained with SEM

The cross section of the SiOC films was obtained with scanning electron microscope SEM. A high resolution image of the SiOC film deposited at RF power level of $350 \mathrm{~W}$ is shown in figure 5 . The $\mathrm{SiOC}$ film seems to possess columnar structure at higher RF power levels.

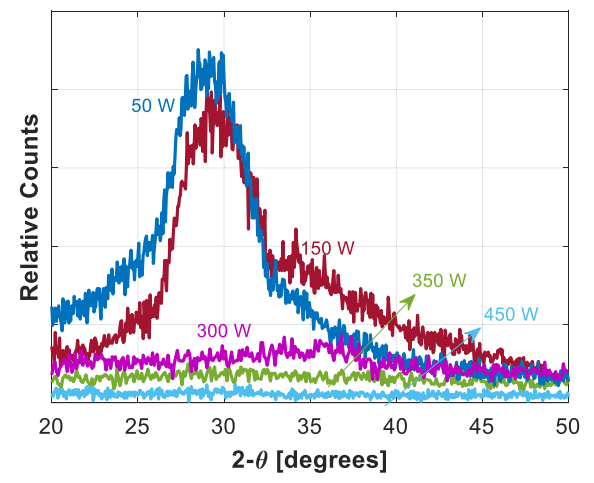

Fig. 6. XRD patterns of SiOC films deposited as a function of RF power $(50-450 \mathrm{~W})$

$\mathrm{X}$-ray diffraction patterns of the $\mathrm{SiOC}$ films deposited as function of RF power $(50-450 \mathrm{~W})$ are shown in figure 6. Since no sharp peaks have been detected in the XRD spectra, the SiOC films seems to be amorphous in phase. The two broad spectral peaks visible at lower RF power levels $(50$ and $150 \mathrm{~W})$ in figure 6 are due to the presence of amorphous silicon dioxide in the SiOC films deposited at lower RF power levels, as they have greater oxygen percentage. The results are in agreement with literature [9].

\section{Conclusion}

In this work, silicon oxycarbide films have been deposited by reactive RF magnetron sputtering technique on Si substrate. The optical properties of the SiOC films deposited as a function of RF power are comprehensively studied with variable angle spectroscopic ellipsometry over the broad UV-Vis-Near IR wavelength range $300-1200 \mathrm{~nm}$. The ellipsometric $\psi$ and $\Delta$ data of the SiOC films have been fitted with lower MSE which demonstrates the accuracy of obtained optical constants. The Kramers-Kronig consistent TaucLorentz model has been used to describe SiOC films and extract its optical parameters. The refractive index $n$ of the deposited SiOC films range from 1.41 to 1.93 at 600 $\mathrm{nm}$ as a function of RF power. The material extinction coefficient $k$ in all the deposited SiOC films has been estimated lower than $10^{-4}$ above $\lambda=1000 \mathrm{~nm}$. In the visible spectrum region, SiOC films also show good transparency. Structural characterization with XRD suggested amorphous phase of the deposited SiOC films. In comparison to CVD [3], SiOC films have been synthesized using sputtering technique with higher deposition rate and lower extinction coefficient $k$ values. Results on SiOC films are promising for integrated photonics applications.

F. A. Memon acknowledges the financial support in his $\mathrm{PhD}$ from European Union under Erasmus Mundus 'LEADERS' project.

\section{References}

1. Grill, Alfred, J. Appl. Phys. 93, 1785-1790 (2003)

2. H. J. Kim, Q. Shao, Y. H. Kim, Surf. and Coat. Tech., 171, 39-45, (2003)

3. S. Gallis, V. Nikas, E. Eisenbraun, M. Huang and A.E. Kaloyeros, J. Appl. Phys. 102, 024302, (2007)

4. R. J. Martín, R. Gago, V. Torres, P. Fernández, U. Kreissig and J.M. Martínez Duart, Thin Sol. Films, 515, 2493-2496, (2006)

5. V. S. Pradeep, M. G. Zajac, R. Riedel and G. D. Soraru, Electrochimica Acta, 119, 78-85, (2014)

6. A. Karakuscu, R. Guider, L. Pavesi, and G. D. Sorarù, J. Amer. Ceram. Soc. 92, 2969-2974, (2009)

7. Sorarù, G. D., D'Andrea, G., Campostrini, R., Babonneau, F. and Mariotto, G., J. Amer. Ceram. Soc., 78, 379-387, (1995)

8. G. Das, P. Bettotti, L. Ferraioli, R. Raj, G. Mariotto, L. Pavesi and G. D. Sorarù, Vib. Spectr., 45, 61-68, (2007)

9. J. V. Ryan and C. G. Pantano, J. Vac. Sc. \& Technology A, 25, 153-159 (2007)

10. P. Du, X. Wang, I.-K. Lin, X. Zhang, Sensors and Actuators A 176, 90-98 (2012)

11. T. Nakai, K. Yusu, Y. Satoh, and S. Ashida. J. Appl. Phys. 45, 1447 (2006)

12. J. I. Larruquert, A. P. Pérez-Marín, S. GarcíaCortés, L. R. Marcos, J. A. Aznárez, and J. A. Méndez, J. Opt. Soc. Am. A 28, 2340-2345 (2011)

13. G. E. Jellison, and F. A. Modine., Appl. Phys. Lett. 69, 371-373 (1996), Erratum, Appl. Phys. Lett. 69, 2137 (1996)

14. Tauc-Lorentz Dispersion formula, Technical note available at www.horiba.com

15. Guide to using $\mathrm{WVASE}^{\circledR}$ spectroscopic ellipsometry data acquisition and analysis 
software, J. A. Woollam Co., Inc., chap.4, p-1620 \& chap. 17, p-21-31 (2012)

16. D. A. G. Bruggeman, Ann. Physik, 24, 636 (1935) 